\title{
EDITORIAL
}

\section{Prologue to the Special Issue on Synthesis}

The Journal of Antibiotics (2013) 66, 105; doi:10.1038/ja.2013.6

S ynthesis of naturally occurring compounds has been a long$\checkmark$ standing traditional source of inspiration and direction for organic chemists worldwide. One important historical application of total synthesis was to clarify any unknown or ambiguous parts of a proposed structure. Another critical function was to confirm the final proposed structure as correct by comparing the isolated natural product with an 'authentic sample' produced by known synthetic methods. However, advanced modern technologies such as NMR spectroscopy, mass spectrometry and X-ray crystallography have become so powerful in solving complex structures that this traditional role for synthesis has been substantially diminished, although not completely eliminated.

However, despite these changes in focus, total synthesis of complex structures still remains an activity of dedicated motivation and inspiration for the development of new reagents, new transformations and new strategies for assembling ever more challenging structures in an efficient and environmentally friendly (green) manner. Furthermore, natural product chemistry has moved more extensively into the arena of biology to elucidate and explain the details involving all of the chemistry that is occurring in biology at the molecular level. Building on its lengthy traditional legacy, total synthesis of natural products still remains an energetic and vibrant force in modern organic chemistry.

This special issue of The Journal of Antibiotics is dedicated to one of those practitioners of total synthesis of complex natural products,
Professor Kuniaki Tatsuta, upon the occasion of his reception of the Ernest Guenther Award in the Chemistry of Natural Products administered by the American Chemical Society. This issue of the journal also celebrates the 102 total syntheses that have been accomplished by Professor Tatsuta during his long career. We congratulate Professor Tatsuta on these lifetime achievements.

In this special issue, Professor Tatsuta has reviewed the 102 total syntheses that have contributed to developing new drugs directly and indirectly. Through each synthetic route that leads to the final product elegantly, we feel and appreciate something artistic. Actually, Professor Tatsuta has often used the word 'art' in his lectures and manuscripts since the time that he studied at Harvard University in the early 1970s directed by Professor RB Woodward who was preeminent in using the word and practicing and illustrating the art of synthesis.

Several of Professor Tatsuta's colleagues and friends have contributed articles to create this special issue of the journal. We wish to acknowledge and sincerely thank the many authors, reviewers and journal personnel whose hard work and dedicated efforts made this special issue possible.

Herbert A Kirst ${ }^{1}$ and Yoshikazu Takahashi ${ }^{2}$ ${ }^{1}$ Section Editor, The Journal of Antibiotics and ${ }^{2}$ Managing Editor, The Journal of Antibiotics E-mail: herbertkirst1@comcast.net 